\title{
Customized Web-based System for Elderly People Using Elements of Artificial Intelligence
}

\author{
František Babič \\ Department of Cybernetics and \\ Artificial Intelligence, Faculty of \\ Electrical Engineering and \\ Informatics, Technical university \\ of Košice, Slovakia \\ frantisek.babic@tuke.sk
}

\author{
Adrián Jančuš \\ Department of Cybernetics and \\ Artificial Intelligence, Faculty of \\ Electrical Engineering and \\ Informatics, Technical university \\ of Košice, Slovakia \\ adrian.jancus@student.tuke.sk
}

\author{
Katarína Melišová \\ Department of Cybernetics and \\ Artificial Intelligence, Faculty of \\ Electrical Engineering and \\ Informatics, Technical university \\ of Košice, Slovakia \\ katarina.melisova@student.tuke.sk
}

\begin{abstract}
Making life easier for the elderly represents a new challenge for the ICT sector. This paper presents a new webbased system designed and implemented with the aim to support the social inclusion and to improve the daily routine of the elderly people within basic information and communication features. The system provides some advanced functionalities to utilise the information value of the data collected within the presented system, e.g. the recommendations based on similar hobbies or health problems; a simple medical diagnostics; a creation of a knowledge base containing experiences and best practices, etc. We designed the system in accordance with local conditions in Slovakia, so its full functioning relies on the progress in e-Health legislation. Presented version is a preliminary result that will be further improved and tested within a real practice.
\end{abstract}

\section{INTRODUCTION}

$\mathrm{P}$ ROGRESSIVE ageing of the population represents a big challenge for various European politics and societies. The European Union has created some initiatives such as Digital Competence or Lifelong Learning Programme (LLP) to support these activities on the national and European level too [7]. Improving the lives of elderly people within suitable ICT solutions in combination with existing social or medical services represents a big challenge for not only public but also the private sector. Successful adaptation of these solutions requires cooperation between all involved organisations, governments, stakeholders and providers. Of course, various local conditions affect the whole implementation process in line with the common EU principles and create the barriers that need to be passed.

Acquired experiences and knowledge from two international EU funded projects OLDES and SPES motivated us to propose a new solution that will meet the identified requirements, will respect existing standards and will be customised for the Slovak local conditions. The project OLDES (Older People's e-services at home) aimed to develop a very low cost and easy to use entertainment and health care platform, designed to ease the life of the elderly in their homes. The implemented platform was tested at two different locations and countries: Prague in the Czech Republic and Bologna in Italy. The SPES project (Support Patients through E-services Solutions) aims at transferring the original approach and results achieved in implementing the OLDES focusing on new target problem domains: dementia, mobility-challenged persons, respiratory problems, and social exclusion [14]. The new tele-health and entertainment platform was tested in four different conditions: Ferrara (Italy), Vienna (Austria), Brno (Czech Republic) and Košice (Slovakia.)

The paper is organised as follows: the introduction with a brief overview of the selected similar approaches or initiatives to identify possible gap for a new solution. The second one describes the proposed solution with emphasis to meet the identified requirements and existing best practices in this domain. Next section presents the current prototype with the results of the testing. The last one concludes the paper and outlines some directions for our future work.

\section{A. State of the Art}

This section presents some selected initiatives and research works with the aim to identify possible gaps or research problems. As we mentioned before, the OLDES project resulted in the solution developed in accordance to the specific needs of the two pilots [13], [15]. The core of this solution is a low-cost PC creating a hub for other connected devices as TV set and the medical devices communicated via Bluetooth. The TV display information provided by the platform and users can access it through a simple remote control. In addition, users can actively participate in discussion groups through adapted handset connected the PC. In the case of medical devices, users tested e.g. a glucometer, scale, adapted version of a sphygmomanometer, etc. After testing partners identified following directions for future improvement: access through the remote controller, prefer medical devices commonly available on the market, the overall architecture of the platform.

The follow-up project SPES aimed to solve these issues and to support four target groups suffered by respiratory problems, dementia, handicapped people and social exclusion. It resulted in an information and communication technology platform connected to the different medical devices and installed in a patients' home [14], [21]. The main advantages of this solution are continuous monitoring of the elderly with the respiratory problems; the presence of various entertainment oriented application that supports orientation and practising memory and a set of services to support the social inclusion of the elderly through virtual communication, information sharing and a personal social network creation. Despite some improvements over the OLDES platform, still, some open issues and gaps existed, 
e.g. SPES platform was available only as a desktop application and this approach required a two-layer transfer of the collected measurements from the medical devices, to the local and main database. From the users point of view, a possibility to create an own personal network was missing, e.g. based on similar hobbies or health problems. In addition, the GUI needed to be refined based on existing trends and best practices. Whereas the currently valid legislation in Slovakia does not allow sharing the medical information between patients and the doctor in the electronic form, some type of decision support system for common health problems can be a very interesting part of the supporting ICT (Information and communication technologies) solution for the elderly.

Decision support systems (DSS) in medicine have a long history that started within a system called Mycin [3]. The actual trend includes a creation of the sufficient quality and broad knowledge base further used for diagnostics of various diseases within suitable analytical methods [16], [12], [11], [6]. These works specifically focus on selected diseases so this factor strongly limits their generality and usability in a wider range. A separate group contains applications called symptom checkers. Typically, they are available as web-based applications and patients have a possibility to start the diagnostics process with an initial description of their symptoms. According to a recent study realised by the Harvard Medical School, most of these sites and applications provide inconsistent and unclear information and patients should not focus on this diagnosis and results [19].

Early diagnosis of the typical civilisation diseases represents one of the main challenges for the cooperation between artificial intelligence represented by analytical methods and medical data obtained through various medical tests or devices [8], [10], [22]. Equally important is a selection of the appropriate attributes for an effective diagnostics [17], [4], [5]. Authors in [1] used data collected in a family practice from Croatia to extract the patient's characteristics necessary for the positive diagnosis of the Metabolic Syndrome. The data include patients in retirement. Authors generated a set of decision tree models within CART or C4.5 algorithms. In addition, authors investigated the optimal cut-off values for the identified key biomarkers.

In summary, we concluded that still some gaps and potential for a new system to support the daily life and health status exist. In close cooperation with a possible target group, based on our previous experiences and actual existing approaches, we identified some features creating the core of a new web-based system. For example a possibility to create an own network of friends without a need to store personal data such as existing social network, a possibility to find a new friend based on similar hobbies or health problems, a possibility to use a simple decision support system to evaluate the discovered symptoms.

\section{WEB-BASED SYSTEM}

\section{A. Functional Requirements}

We specified following requirements based on the provided state of the art analysis, experiences obtained from the SPES project and in communication with a participated group of elderly from the Košice City. Users can use the system on the various devices, i.e. the system will be responsible. Users have a possibility to create their own profile containing information such as a nickname, hobbies, health problems, personal general practitioners or specialist, etc. In additional, users can create their own social network including people recommended based on the similar hobbies or health problems. Through this network, users have a possibility to share various multimedia or news, to use simple on-line chat or to invite their friends to the public or private event. From a medical point of view, users can manage their planned medical examinations, import and visualise the collected measurements from the supported medical devices or use the continuously constructed knowledge base to support the basic medical diagnosis. Last, but not least, the system informs the users about relevant information within simple notifications, current weather forecast or published newspaper articles from selected RSS (Rich Site Summary) source.

\section{B. GUI Proposal}

We designed the graphical user interface (GUI) in accordance to the current relevant W3C standard [22] and verified recommendations [9], i.e. we use a sans serif typeface, $12 \mathrm{pt}$ or $14 \mathrm{pt}$ type size for body text, medium or bold face type. The optimal option for a text alignment is the left. We present the body text in upper and lowercase letters; we use the capital letters and italics in headlines only. We reserve underlining only for the web links. We avoid using a combination of yellow, blue and green colour in a close proximity, because some elderly have problems to discriminate these colours. We used the graphics against a light background. We ensure that users can resize the content without assistive technology up to 200 percent without loss of content or functionality. We provide the labels when content requires a user input. The headings and labels describe topic or purpose.

\section{Architecture Proposal}

Presented prototype is a typical 3-layer client-server model containing a database, back-end and front-end service; see Fig. 1. The analytical package (language R, RStudio) is an integrated module to meet requirements from the legislative and safety point of view. We tested this proof of concept in the conditions in which the elderly mainly verified the predefined scenarios. During the next testing in the larger group of participants, we will discuss relevant lessons learned and possible improvements, not only from the usability point of view but also on the technological side, e.g. to take an advantage of the cloud computing. 


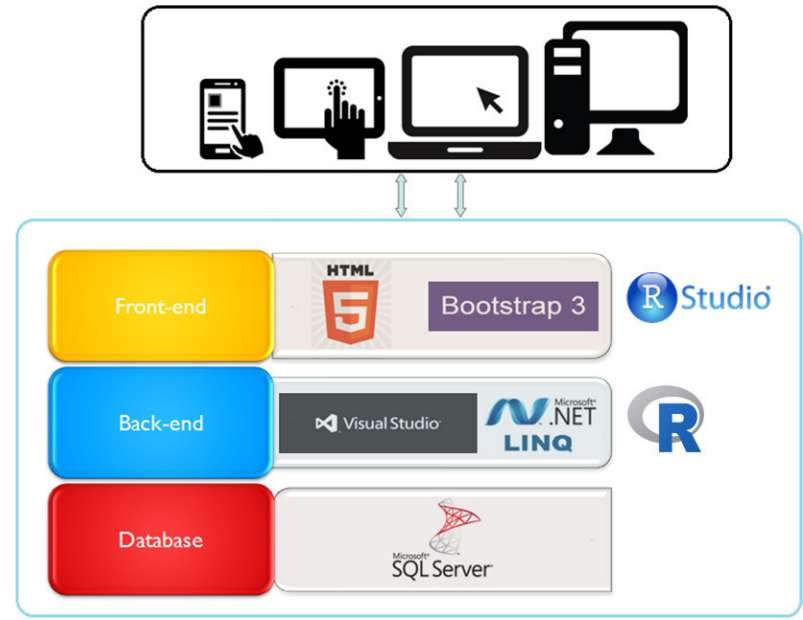

Fig. 1 Architecture with the relevant technologies

\section{Analytical Services Proposal}

Proposed analytical package includes the services necessary for storage, processing, analysis and knowledge base building. We generated initial decision rules and models through free available data samples for selected diseases to show a potential of this type of solution. In real operation, the system will replace this initial data within knowledge relevant to the current users and conditions, i.e. something like self-learning using the methods of artificial intelligence. We organised the whole analytical process in accordance with the CRISP-DM methodology which represents widely accepted approach on how to manage this process effectively [20]. We selected the decision trees based on their ability to process different types of input data, to process data with missing values and the most important factor was a simple understandable visualisation of the generated outputs for people with less knowledge from data mining or machine learning.

We used C4.5 [17] and CART [2] to extract possible interesting rules for diagnostics that were further added to the knowledge base. For example, IF a female is more than 70 years old AND triceps skinfold thickness is more than $28.5 \mathrm{~mm}$ AND the level of insulin is more than $15.15 \mu \mathrm{IU} / 1$ THEN diagnosis of the Mild Cognitive Impairment is positive. Alternatively, IF average level of blood glucose over the previous 3 months was more than 4.4 AND the level of insulin is more than $27.1 \mu \mathrm{IU} / 1$ THEN diagnosis of the Metabolic Syndrome is positive. It is important to say that all included rules need to be verified by cooperated medical experts and it is possible to use them only to support the decision, not as the final diagnosis.

\section{E. Current Prototype}

Tested prototype (available in Slovak language) contained all presented features that were available through a simple and intuitive user environment; see Fig. 2.

Users could read the articles from their favourite web newspapers (Noviny) and watch the weather forecast for the selected location (Počasie). They could create an own profile included hobbies or health problems (Profil) and based

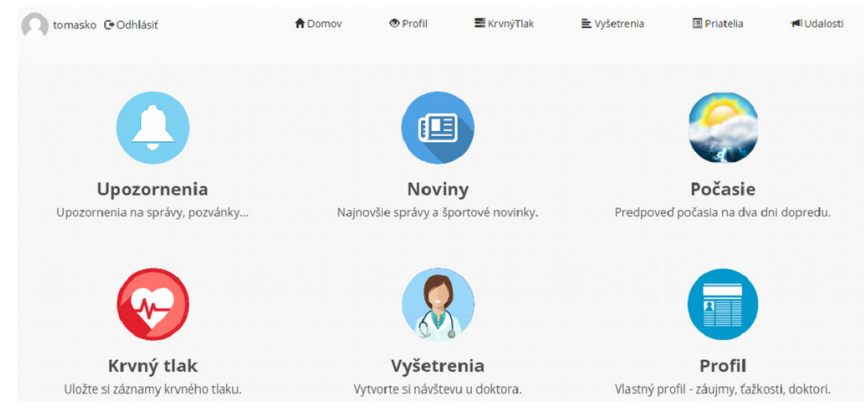

Fig. 2 Main page of the proposed system

recommendation generated from this data find a new friend. In order to improve the health status, they could import their measurements from medical devices (Krvný tlak) with a possibility to visualise (see Fig. 3) and export in the selected format; or create a database containing information about relevant doctors and planned examinations (Vyšetrenia). In addition, a possibility to analyse the collected medical data was at disposal, but the final diagnostics is the responsibility of the general practitioner or specialist.

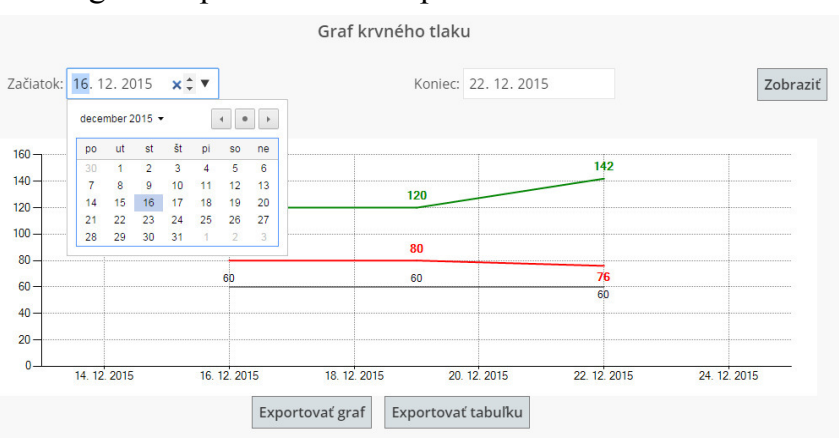

Fig. 3 Visualisation of imported blood pressure measurements

\section{F. Testing}

One of the main reasons why it is not possible to present the proposed system as a finished solution, but only as a proof of concept, is the current legislative in Slovakia covering protection of personal data and e-Health. However, this situation is changing every year under the influence of the various initiatives on the European Union level. We tested our prototype with a small group of elderly in order to evaluate their satisfaction and overall potential of this type of system in Slovak conditions. Elderly realised predefined test scenarios representing typical uses for such systems and answered a predefined questionnaire. This testing confirmed our initial hypotheses such as elderly enjoy the graphical design of the system and it meets relevant requirements and best practices. Also, elderly are satisfied with a set of offered features and with an approach how these features are provided to the users, i.e. the system is easy to use without necessary deep knowledge from the ICT domain. The system helps elderly to reach more active life; to make their daily routine more pleasant and to make their contact with an external environment more intensive. The participated medical expert evaluated the generated models and extracted decision rules based on her knowledge and existing 
literature, e.g. accordance to the IDF (International Diabetes Federation) definition of the Metabolic Syndrome.

\section{CONCLUSION}

The article presents the design and development of the customised web-based system, which would help elderly to the living that is more active and better health status. We aimed to solve some gaps as a possibility to recommend a friend based on similar characteristics or to analyse the data about health status through the simple decision support system. The positive results of the testing confirm our expectations and motivate our future work devoted to the e.g. automatic creation of the user profile based on his behaviour and typical daily habits, or a self-learning and adapting mechanism for created knowledge base. In this case, we will continue to use the methods of artificial intelligence in combination with actual trends in domains as Internet of things, home health care and robotics. In addition, we can understand this version as a proof of concept that creates a good foundation for our future research activities oriented to the $\mathrm{H} 2020$ project proposal.

\section{ACKNOWLEDGMENT}

The work presented in this paper was partially supported by faculty internal research project no. FEI-2015-2 and by the Slovak Grant Agency of the Ministry of Education and Academy of Science of the Slovak Republic under grant No. $1 / 0493 / 16$.

\section{REFERENCES}

[1] F. Babič, L. Majnarič, A. Lukáčová, J. Paralič, A. Holzinger, "On Patient's Characteristics Extraction for Metabolic Syndrome Diagnosis: Predictive Modelling Based on Machine Learning", in Information Technology in Bio- and Medical Informatics, LNCS Vol. 8649, 2014, pp. 118-132, 10.1007/978-3-319-10265-8 11.

[2] L. Breiman, J. H. Friedman, R. A. Olshen, C. J. Stone, "Classification and regression trees", Monterey, CA: Wadsworth \& Brooks/Cole Advanced Books \& Software, 1984.

[3] B. G., Buchanan, E.H. Shortliffe, “ Rule Based Expert Systems: The MYCIN Experiments of the Stanford Heuristic Programming Project" Reading, MA: Addison-Wesley, 1984.

[4] P. Butka, J. Pócs, J. Pócsová, "On Equivalence of Conceptual Scaling and Generalized One-Sided Concept Lattices", in Information Sciences 259, 2017, pp. 57-70, http://dx.doi.org/10.1016/j.ins.2013. 08.047.

[5] P. Butka, J. Pócs, J. Pócsová, "Distributed Computation of Generalized One-Sided Concept Lattices on Sparse Data Tables", in Computing and Informatics 34 (1), 2015, pp. 77-98.

[6] R. J. Conejar, H. K. Kim, "A Medical Decision Support System (DSS) for Ubiquitous Healthcare Diagnosis System", in International Journal of Software Engineering and Its Applications, 8 (10), 2014, pp. 234244, http://dx.doi.org/10.14257/ijseia.20104.8.10.22.

[7] R. Edwards, "Changing Places?: Flexibility, Lifelong Learning and a Learning Society", Routledge, 1997.

[8] P. Han-Saem, C. Sung-Bae, "Evolutionary attribute ordering in Bayesian networks for predicting the metabolic syndrome“, in Expert
Systems with Applications, 39(4), 2012, pp. 4240-4249, 10.1016/j.eswa.2011.09.110.

[9] R. J. Hodes, D. A.B. Lindberg, "Making Your Web Site Senior Friendly", published by the National Institute on Aging and the National Library of Medicine, 2002.

[10] A. Holzinger, M. Dehmer, I. Jurisica, "Knowledge Discovery and Interactive Data Mining in Bioinformatics - State-of-the-Art, in Future challenges and Research Directions", BMC Bioinformatics 15(suppl. 6), I1, 2014, 10.1186/1471-2105-15-S6-I1.

[11] V. A. Kamaev, D. P. Panchenko, N.V. Le, O. A. Trushkina, „An Intelligent Medical Differential Diagnosis System Based on Expert Systems“, in Knowledge-Based Software Engineering, Communications in Computer and Information Science, Volume 466, 2014, pp. 576-584.

[12] T. Matsumoto, Y. Ueda, S. Kawaji, "A software system for giving clues of medical diagnosis to clinician", in Proceedings of 15th IEEE Symposium on Computer-Based Medical Systems (CBMS 2002), IEEE, Maribor, Slovenia, 2002, pp. 65-70, 10.1109/CBMS.2002.1011356.

[13] D. Novák, O. Štepanková, M. Mráz, M. Haluzík, M. Bussoli, M. Uller, K. Maly, L. Nováková, P. Novák, "OLDES: new solution for longterm diabetes compensation management", in Proceedings of 30th Annual International Conference of the IEEE Engineering in Medicine and Biology Society, Vancouver, Canada, 2008, pp. 4346-4349, 10.1109/IEMBS.2008.4650172.

[14] D. Novák, O. Štepánková, S. Rousseaux, M. Busuoli, M. Carulli, G. D’Agosta, T. Gallelli, M. Uller, et al., "Does IT Bring Hope for Wellbeing?", in book Handbook of Research on ICTs for HumanCentered Healthcare and Social Care Services, IGI Global, Editors: Maria Manuela Cruz-Cunha, Maria Miranda, 2103, pp. 270-302.

[15] D. Novák, M. Uller, S. Rousseaux, M. Mráz, J. Smrž, O. Štepanková, M. Haluzík, M. Busuoli, "Diabetes management in OLDES project", in Proceedings of 31 st Annual International Conference of the IEEE Engineering in Medicine and Biology Society, Minneapolis, Minnesota, USA, 2009, pp. 7228-7231, doi: 10.1109/IEMBS.2009. 5335256.

[16] W. Oude Nijeweme-d'Hollosy., L. S. van Velsen, R. Soer, H. J. Hermens, "Design of a web-based clinical decision support system for guiding patients with low back pain to the best next step in primary healthcare", in: Proceedings of the 9th International Joint Conference on Biomedical Engineering Systems and Technologies (BIOSTEC 2016), Rome, Italy, 2016, pp. 229-239, 10.5220/0005662102290239.

[17] J. R. Quinlan, “C4.5: Programs for Machine Learning”, San Mateo: Morgan Kaufmann, 1993.

[18] N. Pérez, M. A. Guevara, A. Silva, I. Ramos, J. Loureiro, "Improving the performance of machine learning classifiers for Breast Cancer diagnosis based on feature selection", in Proceedings of the 2014 Federated Conference on Computer Science and Information Systems, 2014, pp. 209-217, 10.15439/2014F249.

[19] H. L. Semigran, J. A. Linder, C. Gidengil, A. Mehrotra, "Evaluation of symptom checkers for self-diagnosis and triage: audit study", in BMJ, 351:h3480, 2015, 10.1136/bmj.h3480.

[20] C. Shearer, "The CRISP-DM Model: The New Blueprint for Data Mining”, in Journal of Data Warehousing, 5 (4), 2000, pp. 13-22.

[21] G. Tutoky, F. Babic, Wagner J., "ICT-based solution for elderly people", in Proceedings of IEEE 11th International Conference on Emerging eLearning Technologies and Applications, Stará Lesná, Slovakia, 2013, pp. 366-404, 10.1109/ICETA.2013.6674466.

[22] E. Zaitseva, M. Kvassay, V. Levashenko, J. Kostolny, "Introduction to knowledge discovery in medical databases and use of reliability analysis in data mining", in Proceedings of the 2015 Federated Conference on Computer Science and Information Systems, 2015, pp. 311-320, 10.15439/2015F327.

[23] W3C: Web Accessibility Initiative: Developing Websites for Older People: How Web Content Accessibility Guidelines (WCAG) 2.0 Applies. 